\title{
Preface: The Mars Atmosphere and Volatile Evolution (MAVEN) Mission
}

\author{
C.T. Russell ${ }^{1}$ - B.M. Jakosky ${ }^{2}$
}

Published online: 3 November 2015

(C) Springer Science+Business Media Dordrecht 2015

The solar system began in the early solar nebula when the gas and dust accreted to form planetesimals which accumulated into the larger bodies we now know as the planets. Mars, the fourth planet from the sun was less massive than its two neighbors, Jupiter and Earth. But it had grown large enough to differentiate, forming a core and mantle and topped by a crust. It also was surrounded by an atmosphere. This early atmosphere appears to have provided a relatively benign environment, with surficial water that was much more abundant and geologically active than is possible on Mars today. Now, the surface of Mars is cold and dry, and any liquid water will very quickly either freeze or evaporate/sublimate into the atmosphere.

We want to understand how this transition in climate occurred, and what role loss of gas from the atmosphere to space played in this climate change. The records of how the atmosphere evolved are difficult to decipher. We can determine how loss processes operate today, and try to extrapolate backward in time. It is not sufficient to simply use the present day loss rate because the conditions that led to that atmospheric evolution were not the same throughout Mars' history but changed as the Sun evolved. So we must study the loss of atmosphere as a function of solar conditions and apply what we know about the evolution of the Sun to account for these changing conditions and make an accurate estimate of the losses with time. We also can examine characteristics such as the ratios of isotopes of light stable gases and noble gases for clues to the integrated loss to space over time.

A major step in being able to do this was the development of the Mars Atmosphere and Volatile Evolution (MAVEN) mission as part of NASA's Scout program. MAVEN is led by a Principal Investigator, and involves collaboration between the University of Colorado's Laboratory for Atmospheric and Space Physics, the University of California at Berkeley's Space Sciences Laboratory, NASA's Goddard Space Flight Center and Jet Propulsion Laboratory, and Lockheed Martin. While many missions have flown to Mars, none has had such

\footnotetext{
C.T. Russell

ctrussel@igpp.ucla.edu

1 University of California, Los Angeles, CA 90095, USA

2 University of Colorado, Boulder, CO 80303, USA
} 
a comprehensive payload for the study of the upper atmosphere, ionosphere, and solar-wind interactions at Mars and loss of its atmosphere to space. This volume describes the mission and its payload.

The description begins with an overview of the mission by B.M. Jakosky and the MAVEN science team. This paper is followed by the description of the neutral gas and ion mass spectrometer by P.R. Mahaffy and colleagues; this instrument establishes the current composition of the atmosphere and the ionosphere. Ultraviolet emissions are used to map upperatmospheric properties over the globe, and are mapped by the imaging ultraviolet spectrograph described by W.E. McClintock et al. The loss of Mars' atmosphere is controlled in part by the solar wind. MAVEN monitors this with its solar wind ion analyzer described in the next paper by J.S. Halekas et al. The Sun's activity is sporadic and much of the loss of Mars' atmosphere may arise during periods of intense bombardment by solar energetic particles; the measurement of these particles is described by D.E. Larson and colleagues. The ionosphere of Mars consists of both ions and electrons which balance each other's charge. L. Andersson et al. describe the Langmuir probe and waves instrument that measures these electrons and the electrostatic waves associated with their oscillations. The upper ionosphere has ions that are much more energetic than the ions in the lower ionosphere and that represent a significant driver of loss to space. These ions are measured by the suprathermal and thermal ion composition instrument described by J.P. McFadden and colleagues. J.E.C. Connerney and his team describe the magnetometer investigation next; the magnetic-field measurements help us to understand the interactions of the solar wind with the ionosphere, both near and far from crustal magnetic anomalies. The Sun affects the upper atmosphere and ionosphere of Mars not only through particles but also through energetic photons that both heat the atmosphere and drive photochemical and ionospheric processes; F.G. Eparvier and colleagues describe the MAVEN solar extreme ultraviolet monitor that measures this input. It also is possible to monitor the upper atmosphere density structure using its drag effects on spacecraft itself as it orbits through the extremely tenuous upper atmosphere. R.W. Zurek et al. describe how this is achieved by MAVEN's accelerometer and attitude control data. One further instrument, a solar-wind electron analyzer, did not have a description prepared in time for publication in this volume.

In order to make the best use of the MAVEN data, a team of participating scientists was added to the original science team at about the time of launch. The MAVEN project scientist J. Grebowsky and the selected participating scientists describe the plans for this program. The volume concludes with two papers that summarize our current understanding of key areas of MAVEN science, the atmospheric escape from Mars today and through time by R.J. Lillis et al. and the aeronomy of Mars by S.W. Bougher et al. These thirteen articles provide a thorough explanation of what MAVEN does and why.

The success of this volume is due to many people; but first of all, the editors wish to thank the authors who had the difficult job of taking a long history of development of the instruments and mission and distilling it into highly readable articles. The editors also benefited from an excellent group of referees who acted as a test readership, refining the manuscripts provided by the authors. These referees included B.J. Anderson, S. Barabash, L. Didkovsky, T.E. Cravens, E. Dubinin, J.M. Forbes, R. Gladstone, D. Hurley, C. Johnson, P. Kajdič, V. Krasnopolsky, A.F. Nagy, R. Pfaff, P. Richards, J.-A. Sauvaud, S.C. Solomon, J.-E. Wahlund, J.H. Waite, R. Walker, R. Wimmer-Schweingruber, O. Witasse, C. Zeitlin.

Equally important has been the strong support this project has received at Springer and the extra effort expended by Jennifer Satten, Jurgita Vanagaite and Nirmala Kumar. At UCLA we were skillfully assisted by Marjorie Sowmendran who acted as the interface between the authors, referees and the publisher, and at Colorado by Kathleen Cirbo who has supported all of the MAVEN activities. 